\title{
P05- 4 LB. Analysis of virus swarms to identify vaccine immunogens with improved exposure of epitopes recognized by broadly neutralizing antibodies
}

\author{
P Berman*1, SM O'Rourke², B Schweighardt ${ }^{2}$, F Sinangil ${ }^{3}$ and T Wrin ${ }^{2}$
}

\author{
Address: ${ }^{1}$ University of California, Santa Cruz, Santa Cruz, CA, USA, ${ }^{2}$ Monogram Biosciences, South San Francisco, CA, USA and ${ }^{3}$ Global Solutions \\ for Infectious DIseases, South San Francisco, CA, USA \\ * Corresponding author
}

from AIDS Vaccine 2009

Paris, France. 19-22 October 2009

Published: 22 October 2009

Retrovirology 2009, 6(Suppl 3):P396 doi:10.1186/1742-4690-6-S3-P396

This abstract is available from: http://www.retrovirology.com/content/6/S3/P396

(c) 2009 Berman et al; licensee BioMed Central Ltd.

\section{Background}

A major goal of HIV vaccine research is to identify immunogens that elicit broadly neutralizing antibodies (bNAbs). Although many recombinant proteins have been produced that replicate the structure of the envelope protein, none has been able to consistently elicit bNAbs. We now report a new method to identify envelope proteins with rare mutations that improve the exposure of neutralizing sites. These envelopes potentially represent a new source of improved HIV vaccine antigens.

\section{Methods}

Plasma from 28 recent HIV infections were obtained and swarms of HIV envelope genes were amplified by PCR and cloned into an expression plasmid. Pseudovirions were prepared from 24-48 clones per individual and analyzed for infectivity. Subsets of 10 clones selected from each individual were analyzed for sensitivity to neutralization by HIV+ sera possessing bNAbs. Pairs of neutralization sensitive and resistant envelope genes from selected individuals were sequenced and site directed mutagenesis was carried out to identify specific amino acid residues that correlated with neutralization sensitivity.

\section{Results}

Pairs of neutralization sensitive and resistant viruses, with minimal sequence differences, were recovered from 7 of 28 individuals and selected for mutagenesis studies. Analysis of envelope variants from subject 108060 showed a single mutation, Q655R in the C34 helix of gp41, adjacent to the MPER, conferred sensitivity to bNAbs in HIV+ sera, various neutralizing MAbs, CD4-IgG, and Fuzeon. This mutation disrupted a previously unexplored hydrogen bonded ring structure and appears to stabilize the prefusion hairpin intermediate of the 6 helix bundle required for virus fusion. Using this method, we were able to map a novel mutation in the V1/V2 domain of a different individual that conferred sensitivity to bNAbs in HIV+ sera by a distinct mechanism.

\section{Conclusion}

Swarm Analysis is a robust method that provides new insight into neutralization sensitivity and promises to provide a new source of vaccine immunogens. 